\title{
Causa infrecuente de hipo crónico
}

\section{Rare cause of chronic hiccups}

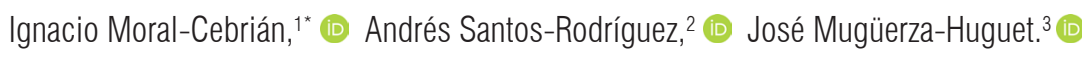

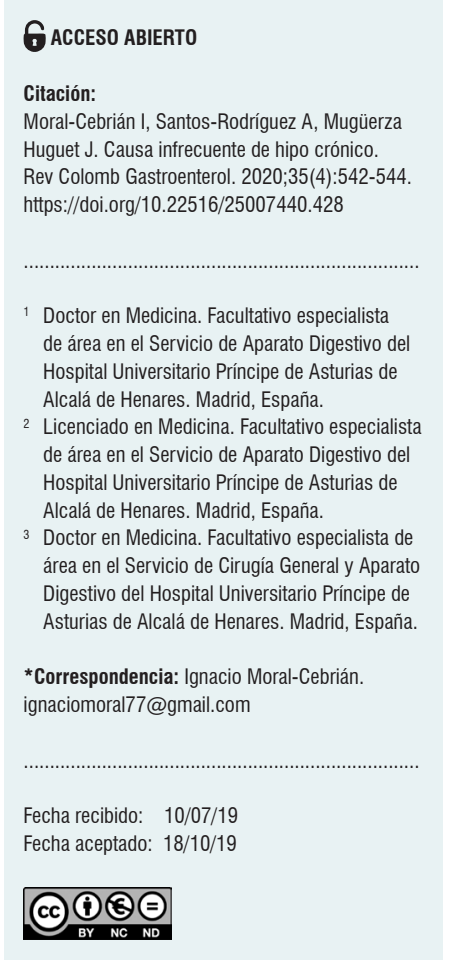

\begin{abstract}
Resumen
El hipo crónico es un síntoma que puede provocar una invalidez significativa y a menudo revela una enfermedad subyacente. A continuación, se presenta el caso de un varón de 68 años que ingresó con hipo de más de 3 meses de duración que se asociaba con epigastralgia, vómitos posprandiales y pérdida ponderal. Había sido intervenido en 2 ocasiones debido a una enfermedad por reflujo gastroesofágico y hernia hiatal, una primera en la que se realizó una fundoplicatura y, posteriormente, una reintervención consistente en el cierre de los pilares diafragmáticos y re-Nissen laparoscópico. La clínica se debía a una obstrucción hiatal por acodamiento de la fundoplicatura previa y fue resuelta mediante la reposición hiatal a los parámetros anatómicos y desmontaje del Nissen previo.
\end{abstract}

\section{Palabras clave}

Hipo, fundoplicatura, hernia hiatal.

\section{Abstract}

Chronic hiccups is a rare symptom that can lead to significant disability and often reveals an underlying disease. The following is the case of a 68 -year-old man who was admitted due to hiccups that had lasted more than 3 months associated with epigastric pain, postprandial vomiting, and weight loss. He had undergone surgery twice due to gastroesophageal reflux disease and hiatal hernia. During the first procedure, a fundoplication was performed, and then, he underwent a reoperation consisting of diaphragmatic pillars closure and laparoscopic Nissen. The symptoms were caused by a hiatal obstruction due to the kinking of the previous fundoplication and were resolved by repositioning the hiatus to anatomical parameters and dismantling the previous Nissen.

\section{Keywords}

Hiccups, Fundoplication, Hiatal hernia.

\section{INTRODUCCIÓN}

El hipo es un síntoma molesto consistente en contracciones mioclónicas, organizadas, involuntarias y repetitivas del diafragma y los músculos intercostales. Este movimiento espasmódico es un fenómeno frecuente y fisiológico a cual- quier edad, al que se suele dar poca importancia o incluso pasar por alto, pero se convierte en una molestia preocupante en algunas situaciones (1).

Se considera crónico si su duración es superior a 48 horas (2). En esos casos puede ser el síntoma de una enfermedad o de una complicación patológica subyacente y suele ser 
refractario al tratamiento. El hipo es considerado intratable cuando se prolonga durante más de un mes y persiste a pesar de 48 horas de tratamiento (3).

Su evolución es la continuación a largo plazo de sacudidas con una frecuencia variable, a menudo con una periodicidad de varios días o semanas al mes y provoca una invalidez significativa. Normalmente, es el síntoma revelador de una enfermedad grave subyacente, por lo que es el único hipo que implica un verdadero tratamiento médico; por esta razón, se debe dar prioridad a la búsqueda de su causa para intentar aplicar un tratamiento etiológico.

\section{CASO CLÍNICO}

Varón de 68 años que acudió a urgencias en octubre de 2018 por persistencia desde hacía más de 3 meses de hipo diario, intermitente, que empeoraba con la ingesta y dificultaba el descanso. Durante dicho período había presentado epigastralgia, náuseas y vómitos posprandiales con pérdida de al menos 4 kilogramos de peso. Su médico de atención primaria había prescrito tratamiento sintomático con Largactil $^{\oplus}$ con escasa mejoría.

El paciente se encontraba en seguimiento en consulta de neumología por asma bronquial en tratamiento con montelukast, fluticasona, salmeterol y bromuro de aclidinio. Debido a una enfermedad por reflujo gastroesofágico y hernia hiatal había sido intervenido en 2 ocasiones, en la primera se le realizó una funduplicatura en abril de 2005 y en la segunda, una reintervención en octubre de 2011 consistente en el cierre de pilares y re-Nissen laparoscópico.

En la exploración llamaba la atención la delgadez del paciente, siendo el resto de la exploración física anodina. El análisis de urgencias mostró leucocitosis, trombocitosis, anemia ferropénica e hipoalbuminemia. Ante la sospecha de patología tumoral se le realizó una tomografía axial computarizada (TAC) abdominal que objetivó un engrosamiento parietal y captación de contraste a nivel de la unión gastroesofágica, y quedó ingresado para completar el estudio.

Se le realizó una gastroscopia en la que se observó abundante líquido en el esófago a pesar de que el paciente guardó más de 10 horas de ayunas, y en el tercio medio y distal había una mucosa con patrón en empedrado blanquecino. Ante la sospecha de candidiasis esofágica, que posteriormente se confirmó, se tomaron biopsias y citología. En la exploración también se observó una hernia hiatal de 3-4 cm con una fundoplicatura que dificultaba el paso del endoscopio sin impedirlo (Figura 1). Además, se analizaron marcadores tumorales que resultaron negativos.

Se trató con fluconazol intravenoso durante un total de 14 días y la clínica mejoró parcialmente luego de las primeras dosis, pero empeoró posteriormente. Por este motivo fue intervenido quirúrgicamente ante la obstrucción hiatal por acodamiento de la fundoplicatura previa (Figura 2). Se le realizó una reposición hiatal a los parámetros anatómicos y se procedió al desmontaje del Nissen previo.

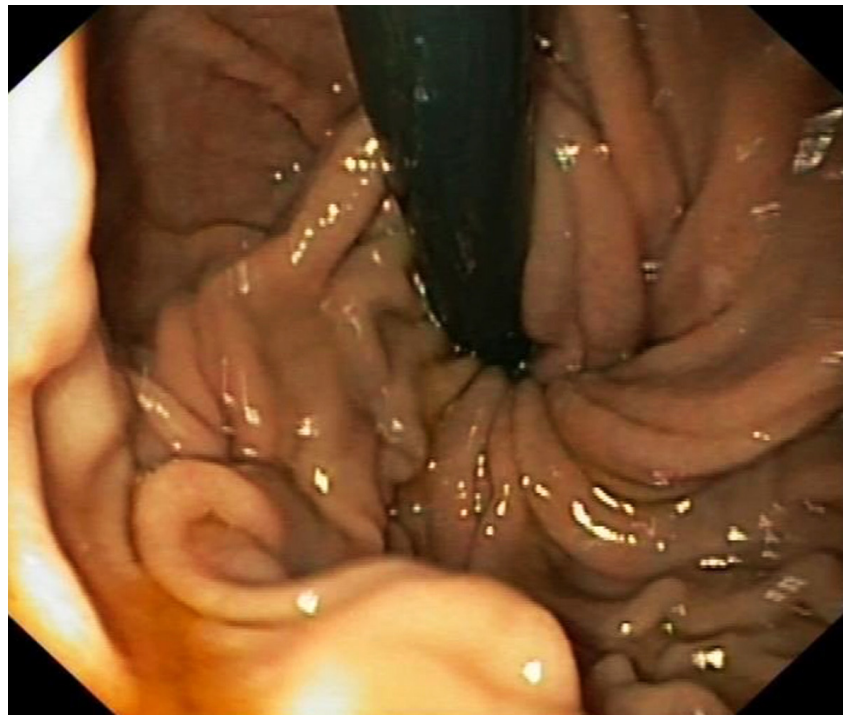

Figura 1. Imagen endoscópica en retroversión de la fundoplicatura durante el ingreso.

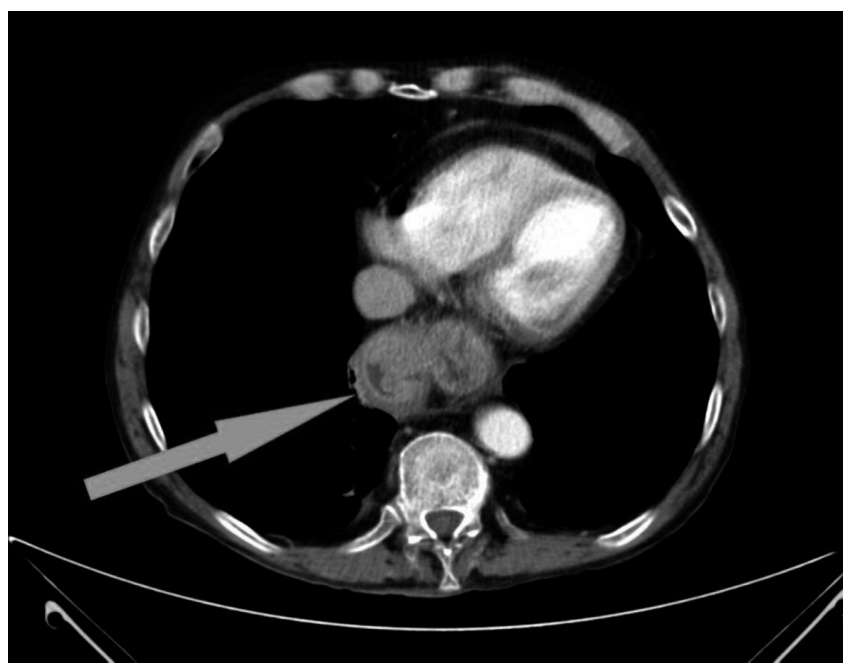

Figura 2. Imagen radiológica de TAC en la que se señala el acodamiento de la fundoplicatura.

La recuperación fue muy satisfactoria y desaparecieron el hipo y las náuseas. En las revisiones posteriores el paciente continúa asintomático y ha ganado peso.

\section{DISCUSIÓN}

Aunque el reflujo gastroesofágico es una entidad frecuente que afecta hasta al $32 \%$ de la población, la aparición de hipo crónico como síntoma de la misma es mucho menos habitual (4). 
La etiología del hipo es numerosa y variada (5). Hay muy pocos estudios que describan la frecuencia de las posibles causas etiológicas del hipo y son muy antiguos $(6,7)$. La mayoría de los episodios agudos son producidos por distensión gástrica, como es el caso de una comida copiosa o de la ingesta de bebidas carbonatadas. La principal causa de hipo crónico es las enfermedades gastroesofágicas, entre las que predomina la esofagitis por reflujo. A continuación, y a gran distancia, están los hipos de causa torácica, abdominal, cerebral e incluso psíquica.

También puede subyacer una causa estructural, infecciosa o inflamatoria que afecte al sistema nervioso central (SNC), al nervio vago, a los nervios frénicos o sus ramas en pacientes con comorbilidad. Es necesario recordar que puede deberse a una enfermedad grave, como una neoplasia o una esclerosis múltiple. El hipo crónico es una entidad algo más frecuente en varones, principalmente si el origen es en el SNC (8).

La resolución de este síntoma pasa por el tratamiento etiológico que lo provoca, como ha sucedido en el caso presentado (9). El paciente presentaba una fundoplicatura que se había desplazado distalmente, volviendo a aparecer una hernia hiatal. La intervención de la hernia hiatal resolvió la sintomatología del paciente. Después de una búsqueda exhaustiva, no hemos encontrado casos publicados de hipo crónico como complicación de una fundoplicatura.

\section{CONCLUSIONES}

Las causas de hipo crónico son muy diversas y es rara su aparición como complicación de una fundoplicatura en los pacientes operados de hernia hiatal. El desmontaje de la fundoplicatura es la solución definitiva para la resolución del hipo crónico de esta etiología.

\section{Fuentes de financiación}

No se ha recibido ayuda financiera para la realización de este artículo.

\section{Conflictos de interés}

El presente artículo no ha sido presentado en ninguna reunión científica.

\section{REFERENCIAS}

1. Cabane J. Hipo crónico: síntomas y diagnóstico. EMC Tratado de medicina 2012;16(1):1-4. https://doi.org/10.1016/S1636-5410(12)61131-3

2. Kolodzik PW, Eilers MA. Hiccups (singultus): review and approach to management. Annal Emerg Med. 1991;20(5):565-73. https://doi.org/ 10.1016/s0196-0644(05)81620-8

3. Hao XT, Wang L, Yan B, Zhou HY. Intractable hiccup caused by spinal cord lesions in demyelination disease. J Spinal Cord Med. 2013;36(6):711-4. https://doi.org/10.1179/2045772313Y.0000000148

4. Rey E, Elola-Olaso CM, Rodríguez-Artalejo, Locke GR 3rd, Díaz-Rubio M. Prevalence of atypical symptoms and their association with typical symptoms of gastroesophageal reflux in Spain. Eur J Gastroenterol Hepatol. 2006; 18(9):969-75. https://doi.org/10.1097/01.meg.0000230081.53298.03

5. Steger M, Schneemann M, Fox M. Systemic review: The pathogenesis and pharmacological treatment of hiccups.
Alimen Pharmacol Ther 2015;42(9):1037-50.

https://doi.org/10.1111/apt.13374

6. Souadjian JV, Cain JC. Intractable hiccup. Etiologic factors in 220 cases. Postgrad Med. 1968;43(2):72-7. https://doi.org/10.1080/00325481.1968.11693139

7. Cabane J, Desmet V, Derenne JP, Similowski T, Launois S, Bizec JL, Orcel B. Chronic hiccups. Rev Med Interne. 1992;13(6):454-9. https://doi.org/10.1016/S0248-8663(10)80030-X

8. Lee GW, Kim RB, Go SI, Cho HS, Lee SJ, Hui D, Bruera E, Kang JH. Gender Differences in Hiccup Patients: Analysis of Published Case Reports and Case-Control Studies. J Pain Symptom Manage. 2016;51(2):278-83. https://doi.org/10.1016/j.jpainsymman.2015.09.013

9. Kohse EK1, Hollmann MW, Bardenheuer HJ, Kessler J. Chronic Hiccups: An Underestimated Problem. Anesth Analg. 2017;125(4):1169-1183. https://doi.org/10.1213/ANE.0000000000002289 